\title{
Introduction of point of care analysis for prescribing warfarin at a Swedish primary care centre
}

Rita Fernholm, Jonas Hermansson

Boo Primary care centre, Stockholm health care services and Centre for evidence based medicine, Stockholm county council, Sweden

\begin{abstract}
Boo Health Centre in Nacka, Sweden, manages approximately 240 patients on warfarin treatment. A risk analysis showed that testing and prescribing of warfarin involved 28 different steps and nine parties, leading to a high risk of errors.
\end{abstract}

The aim of the study was to shorten and simplify the process flow for the testing and prescription of warfarin by introducing point of care analysis (POC). The aim was also to evaluate changes in time expenditure and cost related to the new processes well as the quality in form of time in therapeutic range (TTR) and number of adverse events.

A study with POC was performed during six months in 2014. Time expenditure, cost, TTR, and adverse events related to warfarin treatment were recorded. An evaluation was also conducted in the form of surveys to patients and staff regarding satisfaction with the new process.

The process was shortened from 28 steps and nine parties involved to nine steps and four parties involved. The patient got their test result and met with the prescribing doctor, all within the same visit, meaning that the feedback time for patients was shortened from one to three days by mail to less than 10 minutes at the medical centre. TTR did not change and the incidence of adverse events was not affected. The surveys showed that the overwhelming proportion of patients, doctors, assistant nurses, and laboratory staff were pleased with the changes and the patients would recommend others to monitor their treatment at Boo Health Centre. There was a reduction in time expenditure for the staff. The costs decreased from approximately $8000 € /$ month to about $7000 € /$ month.

The introduction of the POC method enabled a shorter process flow with reduced time expenditure for both patients and staff and reduced costs. TTR did not change. Patients and staff were satisfied with the changes and the patients could take a more active role in their treatment. It is possible that POC analysis may have implications on improved compliance to warfarin treatment, if so, it will increase patient safety.

\section{Problem}

In Sweden warfarin treatment is given either by specialized anticoagulation clinics or by primary health care centres. The quality is consistently high in both settings [1,2] measured as time in therapeutic range (TTR) and as risk of complications.

Boo Health Centre in Nacka, Sweden, has approximately 24000 patients and manages the prescription of warfarin for approximately 240 patients. At the facility 17 general practitioners work. A risk analysis of the warfarin prescriptions was conducted at the primary health care centre. It showed that testing and prescribing involved many different steps and many parties, leading to a high risk of errors. The primary health care centre and the laboratory are two separate entities within the county council, which also presented additional risk elements. The time period between the patient being tested and receiving their prescription by mail was one to three days and the patient was not present when the prescription was made.

Patient safety is the reporting, analysis, and prevention of medical error that often leads to adverse healthcare events. Patients being involved in their care tend to increase patient safety in part through better communication and compliance. Higher patient engagement has shown higher patient safety [3] as well as better patientperceived health outcomes.[4]

The assignment was to test the introduction of a point of care (POC) analysis of INR at the primary care centre. The aim was to cooperate with the Karolinska University Laboratory to shorten and simplify the process flow for the prescription of warfarin in order to simplify the process. Earlier the patient was not present at the time of prescribing but with a POC analysis the patient would be present and could take a more active part in the process. The aim was also to evaluate the quality, time expenditure and cost of the new process flow.

\section{Background}

Long term treatment with oral anticoagulants, such as warfarin or new oral anticoagulants (NOAC), is a very important treatment for stroke prophylaxis in patients with atrial defibrillation (AF), venous thromboembolism, and patients with mechanical heart valves. Warfarin and NOAC use is on the increase worldwide and has potentially serious side effects (eg bleeding or thrombosis). However, warfarin is still the predominate drug.

Warfarin needs closely monitoring of international normalised ratio 
(INR) because it has a narrow therapeutic index and a variation in individual responses; usually INR treatment range is 2.0 to 3.0 . This can cause a problem for as well as patients as for the medical centre that performs the testing and prescribing.

In Sweden more than 250000 patients (approximately $2.5 \%$ of the population) are treated with warfarin or NOAC. Approximately $80 \%$ of them have warfarin. Many new patients who need anticoagulants are given NOAC instead of warfarin but the number of patients with warfarin is still high and need to be managed with the best possible practice.

Point of care INR monitoring has shown to have beneficial impacts on the risk of thromboembolic events, anticoagulation control, patient satisfaction, and quality of life.[5]

\section{Baseline measurement}

Two hundred and forty four patients with warfarin were testing their INR at the primary care centre. Of these, 84 were female and 160 were male, with ages ranging from 31 to 93 years. Most of them had atrial fibrillation. A risk analysis of the warfarin prescriptions was conducted at the primary care centre. It showed that testing and prescribing involved many different steps (28) and many parties (nine), leading to a high risk of errors.

The time period between the patient being tested to receiving their prescription by mail was one to three days. The patient was not present when the prescription was made and did not meet the doctor. There was no communication between the patient and the doctor unless the doctor called the patient up, which seldom happened.

We measured number of reports of adverse events (five reports regarding warfarin treatment) during the period of June to October in 2013 to be able to compare with the same period in 2014 .

TTR was assessed in the 183 patients who were testing their INR both in October 2014 and one year prior at the facility. There was a total of approximately 240 patients getting tested at the primary care centre but of them there was 183 that was also getting tested at the primary care centre one year prior and could be their own controls when TTR was calculated. TTR was 75\% in October 2013. We used a cross section method for percentages of INR in the range.

\section{Design}

A POC method was used to be able to shorten the process as a pilot during six months in 2014 (May to October).

At the start of the study, the Owren method was used at the local laboratory, which is also used at the central laboratory. It is the standard method for the Karolinska University Hospital and for this type of analysis in Sweden. This was an intervention study in which devices for POC analysis were procured in the form of two CoaguChek devices and the process flow was remade. CoaguChek is an evaluated and approved method, [6, 7] although it is more sensitive to interference as the sample is not diluted. The method is also called the dry quick method. The method requires validation toward the Owren method by taking at least two double tests per patient to establish that the patient did not diverse more than 0.3 in INR value between the methods. If a patient has a constant deviation he or she can receive an individualised range in INR when using the CoaguChek method. The period of taking double tests took place January to April 2014 right before the study period.

A doctor was relocated into the laboratory in order to be able to issue warfarin prescriptions directly. A doctor was in the laboratory two hours per day Monday to Friday on a rotating schedule (in our case once every second week). The patients were booked on five minute modules between either $08.00-10.00$ AM or 10.00-12.00 AM. The patient could choose a time that suited them. If they would not consider coming on a booked appointment then they could come another time but in that case the prescription was mailed (only four out of 244 patients chose that).

The study is an improvement study and thereby not requiring ethical approval in accordance to Swedish law. Patients were informed about the POC analysis and could decline to participate with no consequences to their treatment; one patient declined participation on such grounds.

Differences in TTR was evaluated using a chi-2 test in SPSS 20.0.

\section{Strategy}

PDSA cycle 1: The plan was to shorten the number of steps in the process by using a different laboratory test and other changes. We predicted that this would fasten the process and make it safer. Unfortunately, $8 \%$ of the patients were not accepted for the new method because of too much deviation from the standard method (some patients are not suited for the method).[6, 7]

PDSA cycle 2: We establish that it is safer to only have one process. The patients that were not accepted for the method were retested two times and then some could be accepted and some of them received a personalized range for their INR, while others were switched to treatment with NOAC. In this way we could avoid having two parallel processes.

\section{Results}

All patients except one participated in the study. The period of taking double tests resulted in that $8 \%$ of the patients were not accepted for the POC method at the start of the study. The process was shortened from 28 steps to nine, and from nine parties being involved to just four (figure 1).

The time for patients was shortened to less than 10 minutes from arriving to the medical centre, receiving their prescription and being able to go home. The time to analyse with POC was approximately 30 seconds and the prescription was made by a doctor directly after the test was taken. 
For the 183 patients that were testing their INR at the time and one year earlier, TTR was not statistically different ( $p 0.2$ ), from $75 \%$ to $81 \%$ with the POC analysis.

Treatment related adverse events ranged from $1.6 \%$ to $2 \%$ and did not change during the intervention period as compared to the control period.

Surveys were answered by patients ( 180 handed out, 142 answered), doctors (14 handed out, 13 answered), assistant nurses $(\mathrm{n}=5$, all answered) and laboratory staff $(\mathrm{n}=4$, all answered) and a majority of all groups was pleased with the change. $93 \%$ of the patients would recommend others to monitor their treatment at Boo Primary Care Centre, $1 \%$ would not recommend it and $6 \%$ did not answer that question. According to the laboratory staff the patient flow in the laboratory improved.

There was a reduction in time expenditure for the staff in testing and prescribing of warfarin after the introduction of $\mathrm{POC}$ analysis. The associated average time expenditure for the laboratory staff went from three hours and 20 minutes to two hours and 15 minutes per day, for the assistant nurses from five hours to 35 minutes for the group per day. The change in doctor's time expenditure was harder to estimate because instead of many doctors involved it was only one doctor who used two hours per day.

As a result of the reported effects of the intervention, costs associated with the process decreased from approximately 8000 $€ /$ month to about $7000 € /$ month, mostly related to a decrease in time expenditure for the staff.

See supplementary file: ds5529.pptx - "Process flow of warfarin prescription"

\section{Lessons and limitations}

A drawback was that as many as $8 \%$ (19 patients) were not completely suitable for the new method. In previous studies, 1 to $15 \%$ of the patients deviated due to interferences from the standard method Owren [6, 7] depending on how tight the set limits have been. The current limit is that the patient may not deviate more than 0.3 in INR value between CoaguChek and Owren and then $8 \%$ is a level that would still be expected. The limits are currently being reviewed and more patients may be included. Other POC methods will eventually arrive on the market, and perhaps they may be able to include more patients. The 19 patients either were accepted after more tests or received an individualised range in INR if they had a constant deviation $n=9$ or changed from warfarin to NOAC $n=10$ and in that way there was no need for two parallel processes.

\section{Conclusion}

The results of this study show that the introduction of POC testing decreased the number of steps and parties involved in the warfarin prescription process. The results also show that the time expenditure for the staff involved decreased as did the associated costs and the time it took for the patient to receive test result feedback. At the same time, the TTR did not change among the patients with previous test results, and treatment related adverse events did not change.

The introduction of POC analysis appeared to be safe given that treatment related adverse events remained constant and that TTR was similar among 183 patients with previous test results. Patient safety is the reporting, analysis, and prevention of medical error that often leads to adverse healthcare events. Patients being involved in their care tend to increase patient safety in part through better communication and compliance. Higher patient engagement has shown higher patient safety [3] as well as better patient-perceived health outcomes.[4] If the patient receives the prescription in conjunction with the visit, it provides conditions for an individual prescription of warfarin which provides an assured patient and possibly improves treatment compliance.[5] An improved feedback time of INR results could result in patients now being able to directly inform the doctor if they thought a sudden change in their INR was due to some temporary occurrence, such as a forgotten tablet, a change in diet, or temporary use of antibiotics. Such reasons may not necessitate a change in the patient's weekly dose resulting in quality improvements and assured patients.

Similar cost reduction findings have been reported previously also highlighting potential society gains from the use of $\mathrm{POC}$ analysis.[8]

Interestingly, in our area there should be more patients with warfarin treatment, we had only $1 \%$ of our patients who received treatment with warfarin. Given the estimated prevalence of patients requiring anticoagulant treatment, this could indicate that we are treating too few patients. A part of the explanation could be that there are undetected cases of $\mathrm{AF}$ and that some patients in our area managed their treatment at other facilities. Moreover; we had uneven distribution of gender in this study (160 males and 84 women) while other studies have reported a more even gender distribution.[9] Maybe women more often have undetected AF.

In summery the use of POC analysis enabled a completely different process flow which is shorter and with a higher patient engagement. The use of POC analysis also led to reduced time expenditure for both patients and staff and reduced costs at the primary care centre. Patients and staff were satisfied with the changes; thereby the use of $\mathrm{POC}$ analysis may increase treatment compliance in the future. Boo Health Centre has decided to continue with this way of analysing and prescribing warfarin after the test period of six months and we hope that this will spread to more primary care centres in Sweden.

\section{References}

1. Björck $F$, Sandén $P$, Renlund $H$, Svensson $P J$, Själander $A$. Warfarin treatment quality is consistently high in both anticoagulation clinics and primary care setting in Sweden. Thromb Res 2015 Apr 22. pii: S0049-3848(15)00188-7.

2. Arbring K, Uppugunduri S, Lindahl TL. Comparison of prothrombin time (INR) results and main characteristics of patients on warfarin treatment in primary health care centers and anticoagulation clinics. BMC Health Serv Res 2013 Mar 


\section{BMJ Quality Improvement Reports}

7;13:85

3. Weingart SN1, Zhu J, Chiappetta L, Stuver SO, Schneider EC, Epstein AM et al. Hospitalized patients' participation and its impact on quality of care and patient safety Int $\mathrm{J}$ Qual Health Care 2011 June; 23(3): 269-77.

4. Gill PS. Patient engagement: an investigation at a primary care clinic. Int J Gen Med 2013;6:85-98.

5. Point-of-Care International Normalized Ratio (INR) Monitoring Devices for Patients on Long-term Oral Anticoagulation Therapy: An Evidence-Based Analysis.Ont Health Technol Assess Ser 2009;9(12):1-114.

6. SKUP (Scandinavian evaluation of laboratory equipment for primary health care). CoaguChek XS 2007/55: "The main impression of the accuracy of CoaguChek XS is good. No systematic difference between the measurements on CoaguChek XS and the comparison comes forward."

7. Wieloch M, Hillarp A, Strandberg K, Nilsson C, Svensson PJ. Comparison and evaluation of a Point-of-care device (CoaguChek XS) to Owren-type prothrombin time assay for monitoring of oral anticoagulant therapy with warfarin. Thromb Res 2009;7: 344-8.

8. Davidson T, Lindelöf A, Wallén T, Lindahl TL4, Hallert C2. Point-of-care monitoring of warfarin treatment in community dwelling elderly - A randomised controlled study. J Telemed Telecare 2015 Mar 11. pii: 1357633X15574963. [Epub ahead of print]

9. Virjo, I., Mäkelä, K., Aho, J., Kalliola, P., Kurunmäki, H., Uusitalo, L., Ylinen, S. Who receives anticoagulant treatment with warfarin and why? A population-based study in Finland. Scand J Pri Health 2010;28(4), 237-41. doi:10.3109/02813432.2010.514138

\section{Declaration of interests}

Nothing to declare.

\section{Acknowledgements}

We express our gratitude to Lena Pomerleau, Carolina MoultouHeijler, Guje Almroth, Marie L Johansson, and all employees at Boo Medical Centre and to the Karolinska University Laboratory.

\section{Ethical approval}

The work was deemed an improvement study and not a study on human subjects, and local policy meant that ethical approval was not required. Patients were informed about the new quick analysis and could decline to participate. 